\title{
Consejo Mexicano de Cirugía General, A.C. Orígenes y devenir. Presente y futuro
}

\author{
Mexican Board of General Surgery \\ Origin, becoming. Present and future \\ Jordán Zamora-Godínez, Antonio Moreno-Guzmán,* \\ Juan Pablo Pantoja-Millán, ${ }^{*}$ Enrique Jiménez-Chavarría, ${ }^{*}$ Vicente González-Ruiz, ${ }^{*}$ \\ David Velázquez-Fernández, Rafael Humberto Pérez-Soto*
}

Palabras clave: Historia Consejo Mexicano de Cirugía General, cirugía general, certificación.

Keywords: General Surgery Mexican Board

History, general surgery, certification.

${ }^{*}$ Consejo Mexicano de Cirugía General, A.C. México.

Recibido: 26/02/2020 Aceptado: 22/06/2020

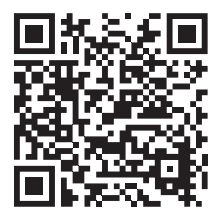

\section{RESUMEN}

Con motivo del cuadragésimo segundo aniversario de la creación del Consejo Mexicano de Cirugía General, A.C. (CMCG) se presenta la siguiente síntesis histórica con la intención de recordar los orígenes de la institución, su evolución y condición actual, y destacar su trascendencia para la cirugía general nacional.

\section{ABSTRACT}

This historical summary was written in honor of the fortysecond anniversary of the creation of the Mexican Board of General Surgery. This summary's objective is remembering the origins of said institution, its evolution, and its current state, as well as, highlighting the relevance it has had for the national general surgery.

\section{INTRODUCCIÓN}

$\mathrm{R}$ especto a los orígenes de la cirugía general en nuestro país, si bien su práctica históricamente se remonta a la época precolombina, fue en el México de finales del siglo XIX y primera mitad del siglo XX cuando se implantaron las primeras especialidades quirúrgicas como la ginecología, urología, cirugía del aparato digestivo, cirugía de la pared abdominal, cirugía ortopédica, oftalmológica, entre otras, y no fue sino hasta el año de 1969 cuando la cirugía general como tal recibió el reconocimiento de especialidad troncal por la División de Estudios de Postgrado de la Facultad de Medicina de la Universidad Nacional Autónoma de México (UNAM). ${ }^{1}$ La formación de los cirujanos anteriores a esta fecha era tutorial y los cirujanos aprendían los fundamentos quirúrgicos con cada una de las rotaciones por los servicios médicos y quirúrgicos de las diferentes especialidades. ${ }^{2}$

En nuestro país, durante la mayor parte de la primera mitad del siglo XX, los médicos "especialistas" eran en buena medida autodidactas, puesto que no existían ni el internado ni las residencias médicas como las conocemos actualmente. Para que los jóvenes médicos interesados en perfeccionar su aprendizaje y orientarlo hacia alguna "especialidad" en particular pudieran hacer realidad su meta, era menester acercarse a uno de sus maestros destacados en la disciplina pretendida. Los maestros eran elegidos por los alumnos generalmente por su carisma y prestigio profesional y los alumnos eran seleccionados o admitidos por el preceptor con base en sus antecedentes como estudiante de pregrado, y en la simpatía que el profesor sintiera por ellos. Debemos recordar que en la década de 1930 solamente

Citar como: Zamora-Godínez J, Moreno-Guzmán A, Pantoja-Millán JP, Jiménez-Chavarría E, González-Ruiz V, VelázquezFernández D, et al. Consejo Mexicano de Cirugía General, A.C. Orígenes y devenir. Presente y futuro. Cir Gen. 2020; 42 (4): 339-344. https://dx.doi.org/10.35366/101406 
existían nueve escuelas de medicina en todo el país, lo que explicaba la cercanía que muchas veces prevalecía en las relaciones maestroalumno en el ámbito de la medicina nacional, puesto que los conocían a profundidad desde su etapa de alumnos de pregrado, de tal manera que ambos sabían perfectamente que podían esperar uno del otro, es decir, era una selección cargada de subjetividad, pero no por ello menos eficiente que la actual. ${ }^{3}$

A partir de 1970 el Programa de Cirugía General se integró formalmente a los cursos de postgrado de la Facultad de Medicina de la UNAM, dicho programa se revisó y actualizó en 1976 y los cursos tuvieron desde ese momento una duración de tres años hasta 1994, cuando el programa se modificó sustancialmente, incrementándose un año al programa inicial, resultando en la actualidad en una duración de cuatro años para los cursos de formación en cirugía general en todo el país, ${ }^{4}$ inclusive en algunas universidades la duración actual de la residencia es de hasta cinco años.

\section{Antecedentes y creación del CMCG}

Como respuesta a las inquietudes de crear el Consejo Mexicano de Cirugía General (CMCG), el 22 de septiembre de 1976 se reunieron en el salón de juntas del Banco de Sangre del Hospital de la Cruz Roja Mexicana los doctores: César Athié Gutiérrez, Guillermo Alamilla Gutiérrez, Carlos Albarrán Treviño, José Luis Bravo Llamosa, Antonio Capetillo Robles Gil, Fernando Díaz Ballesteros, Óscar Díaz Giménez, Enrique Fernández Hidalgo, Enrique Flores Espinosa, Manuel Manzanilla, Carlos Moreno Fernández, Manuel Quijano Narezo, Fernando Romero Castillo, Mario Trápaga Altamirano, José Valencia del Riego, Alfredo Vicencio Tovar y Alberto Villazón Sahagún con el propósito de ratificar los estatutos que regirían el CMCG o/y de iniciar los trámites para su constitución legal ante notario y solicitar su registro ante la Secretaría de Salubridad y Asistencia así como la aprobación de la Academia Nacional de Medicina. Éstos fueron los miembros fundadores del Consejo Mexicano de Cirugía General, A.C., todos ellos profesores titulares de los cursos de especialización en cirugía general registrados en la División de Estudios Superiores de la Facultad de Medicina de la UNAM. ${ }^{5}$

\section{Constitución definitiva del CMCG}

Como parte de los acuerdos de esa reunión, se propuso y aceptó que se enviaran invitaciones para certificarse en un futuro cercano a todos los cirujanos del país, este comunicado fue enviado a través de las instituciones de salud así como de las instituciones formadoras de cirujanos. También fue propuesto y aceptado el logotipo distintivo del CMCG y se acordó invitar a un número igual de cirujanos representantes del interior de la República, es decir, 15 cirujanos, para conferirle el carácter de nacional y se acordó iniciar los trámites notariales correspondientes para legalizar la constitución del CMCG.

Poco más de un año después, el 19 de noviembre de 1977, en el mismo salón de juntas del Banco de Sangre del Hospital de la Cruz Roja Mexicana, se reunieron los 30 cirujanos que a continuación se enumeran:

\begin{tabular}{ll} 
Dr. Guillermo Alamilla Gutiérrez & CDMX* \\
Dr. Carlos Albarrán Treviño & CDMX* \\
Dr. César Athié Gutiérrez & CDMX* \\
Dr. Leonel Barrera Cantú & Chihuahua \\
Dr. José Luis Bravo Llamosa & CDMX* \\
Dr. Antonio Capetillo Robles-Gil & CDMX* \\
Dr. Fernando Díaz Ballesteros & CDMX* \\
Dr. Óscar Díaz Giménez & CDMX* \\
Dr. Enrique Fernández Hidalgo & CDMX* \\
Dr. Enrique Flores Espinoza & CDMX* \\
Dr. Pedro Gama Carpio & Cuanajuato \\
Dr. Gilberto López Betancourt & Nuevo \\
& León \\
Dr. Manuel Manzanilla Sevilla & CDMX* \\
Dr. Armando Martínez Santaella & Oaxaca \\
Dr. Ricardo Mondragón Ballesteros & Estado \\
& de México \\
Dr. Raúl Montalvo Escamilla & Yucatán \\
Dr. Carlos Moreno Fernández & CDMX* \\
Dr. Jaime Paredes Ugarte & Puebla \\
Dr. Javier Preciado Zepeda & Jalisco \\
Dr. Manuel Quijano Narezo & CDMX* \\
Dr. Ricardo Quilantán Antiga & San Luis \\
& Potosí \\
Dr. Gregorio Ramírez Valdez & Coahuila \\
Dr. Fernando Reyes Méndez & Guerrero \\
Dr. Francisco Rivadeneyra & Michoacán \\
\multicolumn{1}{c}{ Hinojosa } & \\
Dr. Rafael Sedas Rendón & Veracruz
\end{tabular}




$\begin{array}{ll}\text { Dr. José Valencia del Riego } & \text { CDMX* } \\ \text { Dr. Juan Vela Trujillo } & \text { Tamaulipas } \\ \text { Dr. Alfredo Vicencio Tovar } & \text { CDMX* } \\ \text { Dr. Alberto Villazón Sahagún } & \text { CDMX* } \\ \text { Dr. Héctor Zazueta Duarte } & \text { Sinaloa }\end{array}$

* Antes D.F.

En esta ocasión, con el objeto de declarar la constitución definitiva del Consejo Mexicano de Cirugía General, A.C., en dicha sesión, el Dr. Manuel Quijano Narezo informó detalladamente los pormenores del registro del CMCG ante la Secretaría de Relaciones Exteriores y la Secretaría de Salubridad y Asistencia. Acto seguido se procedió a elegir al cuerpo de gobierno resultando electos por unanimidad como primer secretario el Dr. Manuel Quijano Narezo, como segundo secretario el Dr. Enrique Flores Espinoza y como tesorero el Dr. Carlos Moreno Fernández. ${ }^{6}$

\section{Devenir}

Durante 1978, primer año de entrada en funciones del CMCG y con base en un artículo transitorio, se certificaron 1,769 cirujanos de todo el país, mismos que, como respuesta a la convocatoria emitida por el CMCG, se acogieron a dicho artículo transitorio y fueron certificados todos con fecha del 11 de noviembre de $1978 .^{7}$

El primer examen de certificación se llevó a cabo los días 7 y 8 de diciembre de 1979 en el tradicional Hospital General de México y a partir de ese año el examen se efectúa anualmente en diferentes sedes.

En la actualidad, el proceso de evaluación se realiza en tres fases: curricular (efectuada altruista y eficientemente por los consejeros), escrita y oral de la manera siguiente: para permitir que los recién egresados de los cursos universitarios puedan presentar el examen escrito días antes de concluir oficialmente su residencia, en el mes de febrero de cada año, mes en que egresan los residentes de sus programas académicos, se lleva a cabo la fase escrita de manera simultánea en cuatro sedes, en las ciudades de Monterrey, N.L., Guadalajara, Jal., Puebla, Pue. y Ciudad de México (Centro de Evaluación de Tlatelolco de la Torre de Vinculación y Gestión Universitaria de la UNAM) y los que resultan aprobados en esa fase escrita presentan la fase oral del examen en el mes de abril para el interior de la República en las sedes de Monterrey, Guadalajara y Puebla y en el mes de mayo, el examen oral se efectúa en la Ciudad de México en las instalaciones del Instituto Nacional de Ciencias Médicas y Nutrición Salvador Zubirán.

Es conveniente mencionar que en el año de 1980 se obtuvo el reconocimiento de la certificación en cirugía general por la Secretaría de Salubridad y Asistencia y en el año de 1981 tanto la Academia Mexicana de Cirugía como el Colegio Americano de Cirujanos otorgaron este reconocimiento. Asimismo, es importante destacar que en el año de 1994 el CMCG firmó el acta constitutiva del Comité Normativo Nacional de Consejos de Especialidades Médicas (CONACEM).

Es de igual relevancia indicar que el $1^{\circ}$ de septiembre de 2011 se modificó la Ley General de Salud en sus artículos 81 y 272-Bis II, en los cuales se establece la obligatoriedad para todos los cirujanos generales en el país de contar con la certificación vigente por el CMCG para poder efectuar procedimientos quirúrgicos de la especialidad. ${ }^{8}$ Asimismo, en el año 2012 el CMCG fue el primer consejo en firmar un convenio con el Instituto Federal de Acceso a la Información (IFAI) dentro del marco de respeto y observancia a la Ley de Protección de Datos Personales.

Cabe destacar que, de común acuerdo con el CONACEM, se han ido abriendo capítulos dentro del mismo CMCG estableciendo los procesos de certificación para los cirujanos bariatras a partir de 2013 y para los cirujanos de trasplante renal a partir de 2014, encontrándose en proyecto el proceso de creación del capítulo de certificación en endoscopia para el cirujano.

Las evaluaciones de certificación y recertificación así como los procesos de recertificación por puntos curriculares que actualmente se efectúan en el CMCG son:

\section{Capítulo de Cirugía General:}

1. Certificación primera vez (escrito y oral).

2. Recertificación por examen.

3. Recertificación por puntos curriculares. 
4. Cirugía para los servicios rurales de salud.

5. Cirujanos con más de 15 años de experiencia.

6. Entrenamiento. Enfatizando que este examen es dirigido a los residentes de tercer año de la especialidad para que se familiaricen con el tipo de exámenes y procesos de inscripción a través de la página web del CMCG, con la finalidad de que su desempeño en el examen de certificación real sea óptimo. Esta modalidad de examen fue implantada en la primera década del presente siglo.

\section{Capítulo de Cirugía Bariátrica:}

1. Certificación primera vez (escrito y oral).

2. Recertificación por examen.

3. Recertificación por puntos curriculares.

Capítulo de Cirugía de Trasplante Renal:

1. Certificación primera vez (escrito y oral).

2. Recertificación por examen.

3. Recertificación por puntos curriculares.

Como parte de los principios que reflejan la filosofía del Consejo Mexicano de Cirugía General, A.C., mencionaremos su misión, visión y valores;

Misión del CMCG: Ser y mantenerse como el único organismo académico certificador en México que lidere la regulación, normatividad y certificación en la cirugía general, contando con el reconocimiento nacional e internacional tanto por la calidad de los procesos de certificación desarrollados como por la calidad y desempeño de su personal y de los miembros del CMCG.

Visión del CMCG: Es el establecimiento, gestión transparente y mejora continua de procesos, parámetros y estándares para efectuar la certificación y recertificación vigente de los cirujanos generales que ejercen su especialidad en la República mexicana, que garanticen la alta y homogénea calidad de conocimientos, habilidades y formación académica de excelencia de todos los egresados en los diversos programas académicos de formación del país.

Valores del CMCG: Responsabilidad, calidad y mejora continua, honestidad, honorabilidad, confiablidad y probidad profesionales. ${ }^{9}$
Con nuestros procesos de certificación buscamos que los cirujanos aprobados se hagan merecedores al aval conferido por el Consejo Mexicano de Cirugía General, A.C. y el Comité Normativo Nacional de Consejos de Especialidades Médicas así como el reconocimiento de idoneidad otorgado tanto por la Academia Nacional de Medicina como por la Academia Mexicana de Cirugía. En virtud de que la vigencia de la certificación es de cinco años, con la recertificación quinquenal buscamos también la constante y óptima actualización del cirujano general para que permanentemente mantenga su nivel de preparación médica.

\section{Lista de los expresidentes del Consejo Mexicano de Cirugía General}
1978-1980
1980-1982
1982-1984
1984-1986
1986-1988
1988-1990
1990-1992
1992-1994
1994-1996
1996-1998
1998-2000
2000-2002
2002-2004
2004-2006
2006-2008
Dr. Manuel Quijano Narezo
Dr. Alberto Villazón Sahagún
Dr. Alfredo Vicencio Tovar
Dr. Jorge Bautista O'Farril
Dr. Óscar Díaz Giménez
Dr. César Gutiérrez Samperio
Dr. Víctor Manuel Arrubarrena Aragón
Dr. Jorge Pérez-Castro
Vázquez
Dr. José Fenig Rodríguez
Dr. Rubén Cortés González
Dr. Ángel Zárate Aguilar
Dr. Alfonso G. Pérez Morales
Dr. Gilberto López Betancourt
Dr. Lorenzo De la Garza
Villaseñor
2008-2011
Dr. Patricio Rogelio Sánchez
Fernández
2008-2011 Dr. Luis Humberto Ortega León
2011-2014 Dra. Adriana Hernández López
2014-2016 Dr. Ricardo Blas Azotla
2016-2018 Dr. Héctor F. Noyola Villalobos
2018-2020 Dr. Jordán Zamora Godínez

\section{Mesa directiva actual (2018-2020)}

Presidente: Dr. Jordán Zamora Godínez

Vicepresidente: Dr. Juan Pablo Pantoja Millán Secretario: Dr. Enrique Jiménez Chavarría Tesorero: Dr. Vicente González Ruiz 


\section{Idoneidad}

Si bien el CMCG se encarga de evaluar a los cirujanos del país, el CMCG a su vez es evaluado por el CONACEM y la Academia Nacional de Medicina y la Academia Mexicana de Cirugía para poder conferirle el reconocimiento de idoneidad avalado por ellos.

Los certificados que se entregan físicamente a los cirujanos que resultan aprobados en los exámenes o en los procesos de recertificación, son elaborados exclusivamente por el CONACEM, cuentan con diversas medidas de seguridad y, muy importante, con el reconocimiento de idoneidad otorgado tanto por CONACEM como por la Academia Nacional de Medicina y la Academia Mexicana de Cirugía. Esta condición de idoneidad y su sello correspondiente debe refrendarse cada cinco años y su obtención depende de la evaluación que el CONACEM y las academias hagan del CMCG en particular. El último reconocimiento de idoneidad fue otorgado al CMCG el 16 de febrero de 2018 y tiene vigencia hasta 2023.

En estos 42 años de existencia del CMCG, tanto el formato del examen como los procesos de inscripción y evaluación han ido evolucionando acorde con la modernidad de cada momento histórico, partiendo de las hojas impresas, pasando por las diapositivas, el uso de hojas ópticas para la calificación y análisis de los resultados, etc., hasta el momento en que se cuenta con una plataforma electrónica muy eficiente que permite tanto el registro e inscripción como la aplicación de exámenes y evaluación de los mismos.

Toda la información se encuentra integrada en una base de datos confiable vinculada además con la página web del CMCG (www. cmcgac.org.mx) a través de la cual los sustentantes realizan el proceso de inscripción y en la que, entre otras cosas, se publican las convocatorias para los diferentes tipos de examen ofertados por el CMCG y el directorio de médicos con certificación vigente, mismos que pueden ser consultados por cualquier persona, en cualquier momento $y$ en cualquier parte del mundo.

En este mismo periodo el CMCG ha certificado, en al menos una ocasión, a 10,232 cirujanos generales, de los cuales actualmente 4,242 cuentan con certificación vigente.

\section{Sedes del CMCG}

El primer domicilio del CMCG estuvo ubicado en Av. Veracruz No. 93-202, Colonia Condesa, Delegación Cuauhtémoc, México, D.F., primero en renta y a partir de 1990 en propiedad.

Domicilio actual y propio desde agosto de 2011: World Trade Center de la Ciudad de México, calle Montecito No. 38, Piso 18, Oficina 21, Colonia Nápoles, Alcaldía Benito Juárez, Ciudad de México, C.P. 03810. Teléfonos 55 5286-3012 y 55 5211-0074.

\section{CONCLUSIÓN}

En resumen, la formación de especialistas médicos en el país está integrada por una gran cantidad de instituciones, inicialmente públicas y a partir del último tercio del siglo pasado también de instituciones privadas, con diversos grados de desarrollo y por ende, con distintas ofertas educativas, por lo que los especialistas egresados tienen gran heterogeneidad y diferencias en su formación. ${ }^{10}$ A pesar de ello, este sistema es hasta el momento la mejor de las opciones para la formación de recursos humanos para la salud.

Ahora bien, es precisamente debido a esta heterogeneidad en la formación del cirujano general en México que se hace indispensable contar con un organismo académico, profesional y completamente neutral que se encargue de establecer los criterios académicos y deontológicos mínimos con los que debe contar un cirujano general, independientemente de la universidad que lo avale o del hospital u hospitales donde se haya formado y de evaluar, por medio de sus exámenes, a todos los cirujanos del país con la finalidad de acreditar que el cirujano certificado vigente cuenta con la preparación adecuada para que sus pacientes reciban una atención de calidad en cualquier parte del territorio nacional en las instituciones de salud tanto públicas como privadas, y ese organismo es precisamente el Consejo Mexicano de Cirugía General, A.C. 


\section{REFERENCIAS}

1. De la Garza Villaseñor L. De la cirugía y su enseñanza en México. Reseña histórica, 1325-2000. Rev Inv Clin. 2003; 55: 719-735.

2. León López G. Prólogo. En: León López G. Historia de la Cirugía General en la República Mexicana. Vol. I. Asociación Mexicana de Cirugía General A.C. México: Ed. Graphimedic S.A. de C.V.; 2011. p. 11.

3. Ortiz Monasterio F. La enseñanza médica de posgrado durante los últimos 40 años. En: Sepúlveda B. La evolución de la medicina en México durante las últimas cuatro décadas. Conmemoración del cuadragésimo aniversario de la fundación de El Colegio Nacional. México: El Colegio Nacional; 1984. p. 74.

4. Carrasco Rojas JA. Cirugía general. En: Fajardo Dolci G, Graue Wiechers E, Kershenobich Stalnikowitz D, Vilar Puig P. Desarrollo de las especialidades médicas en México. Secretaría de Salud, Universidad Nacional Autónoma de México, Academia Nacional de Medicina. México: Ed. Alfil S.A. de C.V.; 2012. p. 83.

5. Acta de la sesión inaugural del Consejo Mexicano de Cirugía General, A.C., del 22 de septiembre de 1976.

6. Acta Constitutiva de la sesión del Consejo Mexicano de Cirugía General, A.C., del 19 de noviembre de 1977.

7. Zermeño-Gómez MG, Kobeh-Jirash JA, MorenoGuzmán A, Jiménez-Chavarría E, Pantoja-Millán JP, Noyola-Villalobos H. La Certificación en Cirugía
General a 42 años de la fundación del Consejo Mexicano de Cirugía General. Cir Gen. 2019; 41: 314-321.

8. Diario Oficial de la Federación. 01/09/2011. [Consultado el 3 de enero de 2020]. Disponible en: http://www.dof.gob.mx/nota_detalle.php? codigo $=52$ 07454\&fecha $=01 / 09 / 2011$

9. Consejo Mexicano de Cirugía General, A.C. [Consultado el 6 de diciembre de 2019]. Disponible en: www.cmcgac.org.mx

10. Vargas Saldo E. La formación de médicos especialistas. En: Vázquez Martínez D, Cuevas Álvarez R, Crocker Sagástume R. La formación de personal de salud en México. Programa Colaborativo de Recursos Humanos en Salud, Instituto de Investigación de Recursos Humanos en Salud. México: Centro Universitario de Ciencias de la Salud de la Universidad de Guadalajara, AMFEM y UAM-Xochimilco; 2005. p. 118.

Correspondencia:

\section{Dr. Jordán Zamora Godínez}

World Trade Center, Montecito Núm. 38,

Piso 18, Oficina 21, Col. Nápoles, 03810,

Alcaldía Benito Juárez, Ciudad de México.

Teléfonos: 55 5286-3012 y 55 5211-0074

E-mail: cmcgac@live.com y

jordanzamoragodinez@gmail.com 